\title{
Some identities for derangement and Ward number sequences and related bijections
}

\author{
DAvid CALlan \\ Department of Statistics \\ University of Wisconsin \\ Madison, WI 53706, USA \\ email: callan@stat.wisc.edu \\ and \\ TOUfIK MANSOUR \\ Department of Mathematics \\ University of Haifa \\ 3498838 Haifa, Israel \\ email: tmansour@univ.haifa.ac.il \\ and \\ MARK ShatTUCK \\ Department of Mathematics \\ University of Tennessee \\ Knoxville, TN 37996 USA \\ email: shattuck@math.utk.edu
}

(Received: January 7, 2016, and in revised form April 23, 2016.)

\begin{abstract}
We establish an alternating sum identity for three classes of singleton-free set partitions wherein the number of elements minus the number of blocks is fixed: (i) permutations, that is, partitions into cycles, (ii) unrestricted partitions, and (iii) contents-ordered partitions. Both algebraic and combinatorial proofs are given, the latter making use of a sign-changing involution in each case. As a consequence, combinatorial proofs are found of specific cases of recent identities of Gould et al. involving both kinds of Stirling numbers.
\end{abstract}

Mathematics Subject Classification(2010). 05A19, 05A15.

Keywords: derangement, Ward numbers, Stirling numbers, combinatorial proof.

\section{Introduction}

A derangement is a permutation having no cycles of length one. The number $d(n)$ of derangements of $[n]=\{1,2, \ldots, n\}$ is given by sequence A000166 in OEIS [9]. Consider the refinement $d(n, k)$ counting the derangements of $[n]$ having exactly $k$ cycles. The sequence $d(n, k)$ occurs as entry A008306 in OEIS [9]. Here, we prove a new property of these numbers which also holds for a polynomial generalization. We then consider the analogous problem for set partitions and Lah distributions (i.e., 
contents-ordered partitions): let $e(n, k)$ denote the number of $k$-block partitions of $[n]$ in which no block is a singleton and, similarly, let $f(n, k)$ be the number of $k$-block Lah distributions having no singleton blocks. The sequences $e(n+k, k)$ and $f(n, k)$ occur as entries A134991 and A076126 in [9], respectively.

In this paper, we evaluate the sum

$$
S_{u}(n)=\sum_{k=1}^{n}(-1)^{n+k} u(n+k, k),
$$

when $u(n, k)=d(n, k), e(n, k)$, or $f(n, k)$. Due to the singleton-free restriction, this sum is over all the respective partitions for which \# elements $-\#$ blocks $=n$. In each case, we provide both algebraic and combinatorial proofs of the result, for the latter making use of sign-changing involutions (in the spirit of [1, 2]). We remark that it does not appear to be the case that the sum $S_{u}(n)$ has a simple closed form when one considers instead the analogous counting functions for all permutations, partitions, or Lah distributions having a fixed number of cycles or blocks. One reason for this may be that it is only under the restriction of no singletons that the summation range $k=1$ to $n$ covers all nonzero values of the summand.

The organization of this paper is as follows. In the next section, we consider the case when $u(n, k)=d(n, k)$ in the problem above and also give a polynomial generalization of the result. In the third section, we provide algebraic proofs of the analogous results for $e(n, k)$ and $f(n, k)$. Also, a pair of related identities involving the Stirling numbers of both kinds (corresponding to the $m=1$ case of a more general algebraic result of Gould et al. [5]) is afforded a bijective proof. In the final section, combinatorial proofs are given of our results for $e(n, k)$ and $f(n, k)$. For the former case, we represent the blocks of a singleton-free set partition, graphically, as two horizontal rows and pair all partitions except those corresponding to a perfect matching in the bipartite graph $[n] \times[n+1,2 n]$ according to the position of a certain violating block. For the latter case, a close connection is made between contents-ordered set partitions enumerated by $f(n, k)$ and "marked" lattice paths wherein a subset of the vertices incident with two upsteps is distinguished.

\section{Identity for derangement numbers}

Let $d(n, k)$ denote the number of derangements of $[n]$ containing exactly $k$ cycles. Considering whether or not the cycle containing the element $n$ is of length two, and if so, the other member of $[n]$ within that cycle, leads to the recurrence

$$
d(n, k)=(n-1) d(n-2, k-1)+(n-1) d(n-1, k), \quad n, k \geq 1,
$$

with $d(0,0)=1$. Note that $d(n, k)=0$ if $n \leq 2 k-1$. We consider the polynomial generalization of $d(n, k)$ given by

$$
d_{a, b}(n, k)=a(n-1) d_{a, b}(n-2, k-1)+b(n-1) d_{a, b}(n-1, k), \quad n, k \geq 1,
$$

with $d_{a, b}(0,0)=1$, where $a$ and $b$ are indeterminates.

Define the generating functions

$$
D_{k}(x ; a, b)=\sum_{n \geq 2 k} d_{a, b}(n, k) x^{n}, \quad k \geq 0,
$$


and

$$
J_{k}(x ; a, b)=\sum_{n \geq 2 k} d_{a, b}(n, k) \frac{x^{n}}{n !}, \quad k \geq 0,
$$

with $D(x, y ; a, b)=\sum_{k \geq 0} D_{k}(x ; a, b) y^{k}$ and $J(x, y ; a, b)=\sum_{k \geq 0} J_{k}(x ; a, b) y^{k}$. Then recurrence (2) can be written as

$$
D_{k}(x ; a, b)=a x^{2} \frac{d}{d x}\left(x D_{k-1}(x ; a, b)\right)+b x^{2} \frac{d}{d x} D_{k}(x ; a, b), \quad k \geq 1,
$$

which implies

$$
\left(1-a x^{2} y\right) D(x, y ; a, b)=1+x^{2}(b+a x y) \frac{d}{d x} D(x, y ; a, b) .
$$

In particular, letting $y=-\frac{b}{a x}$, we obtain $D\left(x,-\frac{b}{a x} ; a, b\right)=\frac{1}{1+b x}=\sum_{n \geq 0}(-b)^{n} x^{n}$. Hence,

$$
\begin{aligned}
\sum_{n \geq 0}(-b)^{n} x^{n} & =\sum_{k \geq 0} D_{k}(x ; a, b) \frac{(-b)^{k}}{(a x)^{k}} \\
& =\sum_{k \geq 0} \sum_{n \geq 2 k} d_{a, b}(n, k) x^{n} \frac{(-b)^{k}}{(a x)^{k}} \\
& =\sum_{k \geq 0} \sum_{n \geq 0} d_{a, b}(n+k, k) x^{n} \frac{(-b)^{k}}{a^{k}} \\
& =\sum_{n \geq 0} x^{n} \sum_{k=0}^{n} d_{a, b}(n+k, k) \frac{(-b)^{k}}{a^{k}}
\end{aligned}
$$

where we have used $d_{a, b}(n+k, k)=0$ when $2 k>n+k$. Thus, we can state the following result.

THEOREM 2.1 For all $n \geq 1$,

$$
\sum_{k=1}^{n} \frac{(-b)^{k}}{a^{k}} d_{a, b}(n+k, k)=(-b)^{n} .
$$

If instead we use the exponential generating function, then recurrence (2) can be rewritten as

$$
\frac{d}{d x} J_{k}(x ; a, b)=a x J_{k-1}(x ; a, b)+b x \frac{d}{d x} J_{k}(x ; a, b), \quad k \geq 1,
$$

with $J_{0}(x ; a, b)=1$. Thus, we obtain

$$
J_{k}(x ; a, b)=\frac{a^{k}(-b x-\ln (1-b x))^{k}}{b^{2 k} k !}, \quad k \geq 0 .
$$

This can be shown by inducting on $k$, the $k=0$ case obvious. If $k \geq 0$, then we have by (5) and the induction hypothesis,

$$
\begin{aligned}
J_{k+1}(x ; a, b) & =a \int_{0}^{x} \frac{t}{1-b t} J_{k}(t ; a, b) d t=\frac{a^{k+1}}{b^{2 k} k !} \int_{0}^{x} \frac{t}{1-b t}(-b t-\ln (1-b t))^{k} d t \\
& =\frac{a^{k+1}}{b^{2 k+2} k !} \int_{0}^{x}\left(-b+\frac{b}{1-b t}\right)(-b t-\ln (1-b t))^{k} d t \\
& =\frac{a^{k+1}}{b^{2 k+2}(k+1) !}(-b x-\ln (1-b x))^{k+1}
\end{aligned}
$$


which completes the induction.

Corollary 2.2 We have

$$
\sum_{k \geq 0} \frac{1}{b^{k} k !} \frac{d^{k}}{d x^{k}}(b x+\ln (1-b x))^{k}=e^{-b x}
$$

Proof. By (4), we obtain

$$
e^{-b x}=\sum_{n \geq 0} \frac{(-b)^{n} x^{n}}{n !}=\sum_{n \geq 0} \frac{x^{n}}{n !} \sum_{k=0}^{n} \frac{(-b)^{k}}{a^{k}} d_{a, b}(n+k, k)=\sum_{k \geq 0} \frac{(-b)^{k}}{a^{k}} \frac{d^{k}}{d x^{k}} J_{k}(x ; a, b),
$$

which implies (6)

We conclude this section by giving a bijective proof of the $a=b=1$ case of (4):

$$
\sum_{k=1}^{n}(-1)^{k} d(n+k, k)=(-1)^{n}, \quad n \geq 1 .
$$

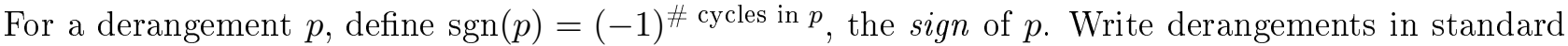
cycle form: largest entry first in a cycle, with first entries increasing left to right, as in

$$
31 / 624 / 75 / 98 \text {. }
$$

Note that $21 / 43 / 65 / \cdots / 2 n 2 n-1$ is then special: it is the only derangement of $[2 n]$ consisting of doubletons whose differences are all 1 .

Let $\mathcal{D}_{n, k}$ denote the set of derangements of $[n]$ with $k$ cycles. Set

$$
\mathcal{A}_{n}=\left(\bigcup_{k=1}^{n} \mathcal{D}_{n+k, k}\right) \backslash\{21 / 43 / 65 / \cdots / 2 n 2 n-1\},
$$

where each element of $\mathcal{A}_{n}$ has a sign as defined above. Since the derangement excluded from $\mathcal{A}_{n}$ has sign $(-1)^{n}$, the identity (7) is equivalent to

$$
\sum_{p \in \mathcal{A}_{n}} \operatorname{sgn}(p)=0 .
$$

To establish (9), we define a sign-reversing involution on $\mathcal{A}_{n}$ as follows. For $p \in \mathcal{A}_{n}$, first scan the cycles right to left. As long as they are doubletons with maximum possible first entry and difference 1 , erase them, and record the number of erased doubletons. (Note that after this initial step, a nonempty derangement will always be left, because $21 / 43 / 65 / \cdots / 2 n 2 n-1$ is excluded from $\mathcal{A}_{n}$.) For example (8), we get

$$
31 / 624 / 75
$$

with one erased doubleton. If the last cycle is now a doubleton (as here), delete it and insert its second entry in the second position of the previous cycle. Here, we get $31 / 6524$. On the other hand, if it is not a doubleton, as in $432 / 651$, delete the second entry of the last cycle, say $a$, and append a new doubleton $m+1 a$, where $m$ is the first entry of the last cycle. Here we get $432 / 61 / 75$. Finally, append doubletons with difference 1 to match the number of erased doubletons. Example (8) thus leads to $31 / 6524 / 87$.

This mapping preserves $\mathcal{A}_{n}$, is an involution, and always changes the number of cycles by 1 . Thus it serves as the desired sign-reversing involution. 


\section{Analogues for partitions}

Let $e(n, k)$ denote the number of partitions of $[n]$ having $k$ blocks and no singletons. Members of the array $\{e(n+k, k): 1 \leq k \leq n\}$ are known as Ward numbers; see, e.g., [3, 4, 11]. The $e(n, k)$ satisfy the recurrence

$$
e(n, k)=(n-1) e(n-2, k-1)+k e(n-1, k), \quad n, k \geq 1,
$$

with $e(0,0)=1$ and $e(n, k)=0$ if $n \leq 2 k-1$, upon considering whether or not the element $n$ belongs to a doubleton block.

THEOREM 3.1 For all $n \geq 1$,

$$
\sum_{k=1}^{n}(-1)^{n+k} e(n+k, k)=n ! .
$$

Proof. Let $g(n, k)=e(n, k) /(n-k)$ !. Then (10) can be expressed as

$$
(n-k) g(n, k)=(n-1) g(n-2, k-1)+k g(n-1, k),
$$

with $g(0,0)=1$ and $g(n, k)=0$ if $n \leq 2 k-1$. Define $E_{k}(x)=\sum_{n \geq 2 k} g(n, k) x^{n-k}$. Then, by multiplying the above recurrence by $x^{n-k-1}$ and summing over $n \geq 2 k$, we obtain

$$
\frac{d}{d x} E_{k}(x)=\frac{d}{d x}\left(x E_{k-1}(x)\right)+(k-1) E_{k-1}(x)+k E_{k}(x), \quad k \geq 1,
$$

with $E_{0}(x)=1$.

Now, define $E(x, y)=\sum_{k \geq 0} E_{k}(x) y^{k}$. Then

$$
\frac{d}{d x} E(x, y)=y \frac{d}{d x}(x E(x, y))+y(1+y) \frac{d}{d y} E(x, y) .
$$

Thus, $\frac{d}{d x} E(x,-1)=-\frac{d}{d x}(x E(x,-1))$, which, since $E(0,-1)=1$, implies that $E(x,-1)=1 /(1+x)$. Hence,

$$
\begin{aligned}
\sum_{n \geq 0} x^{n} & =\frac{1}{1-x}=E(-x,-1) \\
& =\sum_{k \geq 0} \sum_{n \geq 2 k} g(n, k)(-x)^{n-k}(-1)^{k}=\sum_{k \geq 0} \sum_{n \geq 2 k} e(n, k) \frac{(-x)^{n-k}(-1)^{k}}{(n-k) !} \\
& =\sum_{k \geq 0} \sum_{n \geq k}(-1)^{n+k} e(n+k, k) \frac{x^{n}}{n !}=\sum_{n \geq 0} \sum_{k=0}^{n}(-1)^{n+k} e(n+k, k) \frac{x^{n}}{n !}
\end{aligned}
$$

A comparison of the coefficients of $x^{n}$ completes the proof.

Let $f(n, k)$ denote the number of contents-ordered partitions of $[n]$ having $k$ blocks and no singletons. Considering whether or not the block containing $n$ is a doubleton leads to the recurrence

$$
f(n, k)=2(n-1) f(n-2, k-1)+(n+k-1) f(n-1, k), \quad n, k \geq 1,
$$

with $f(0,0)=1$ and $f(n, k)=0$ if $n \leq 2 k-1$. 
Theorem 3.2 For all $n \geq 1$,

$$
\sum_{k=1}^{n}(-1)^{n+k} f(n+k, k)=(n+1) !
$$

Proof. Let $h(n, k)=f(n, k) /(n-k)$ !. Then (12) can be expressed as

$$
(n-k) h(n, k)=2(n-1) h(n-2, k-1)+(k+n-1) h(n-1, k),
$$

with $h(0,0)=1$ and $h(n, k)=0$ if $n \leq 2 k-1$. Let $F_{k}(x)=\sum_{n \geq 2 k} h(n, k) x^{n-k}$. Multiplying the above recurrence by $x^{n-k-1}$, and summing over $n \geq 2 k$, yields

$$
(1-x) \frac{d}{d x} F_{k}(x)=2 \frac{d}{d x}\left(x F_{k-1}(x)\right)+2(k-1) F_{k-1}(x)+2 k F_{k}(x), \quad k \geq 1,
$$

with $F_{0}(x)=1$.

Now, define $F(x, y)=\sum_{k \geq 0} F_{k}(x) y^{k}$. Then

$$
(1-x) \frac{d}{d x} F(x, y)=2 y \frac{d}{d x}(x F(x, y))+2 y(1+y) \frac{d}{d y} F(x, y) .
$$

Thus, $(1-x) \frac{d}{d x} F(x,-1)=-2 \frac{d}{d x}(x F(x,-1))$, which, since $F(0,-1)=1$, implies that $F(x,-1)=$ $1 /(1+x)^{2}$. Hence,

$$
\begin{aligned}
\sum_{n \geq 0}(n+1) x^{n} & =\frac{1}{(1-x)^{2}}=F(-x,-1) \\
& =\sum_{k \geq 0} \sum_{n \geq 2 k} h(n, k)(-x)^{n-k}(-1)^{k}=\sum_{k \geq 0} \sum_{n \geq 2 k} f(n, k) \frac{(-x)^{n-k}(-1)^{k}}{(n-k) !} \\
& =\sum_{k \geq 0} \sum_{n \geq k}(-1)^{n+k} f(n+k, k) \frac{x^{n}}{n !}=\sum_{n \geq 0} \sum_{k=0}^{n}(-1)^{n+k} f(n+k, k) \frac{x^{n}}{n !} .
\end{aligned}
$$

A comparison of the coefficients of $x^{n}$ completes the proof.

Let $c(n, k)$ and $S(n, k)$ denote the Stirling numbers of the first and second kind defined, respectively, by the recursions $S(n, k)=S(n-1, k-1)+k S(n-1, k)$ and $c(n, k)=c(n-1, k-1)+(n-1) c(n-1, k)$ for $k \geq 1$, with $S(n, 0)=c(n, 0)=\delta_{n, 0}$ for $n \geq 0$. See, e.g., [6, Section 6.1], [7] or [10, Sections 1.3-1.4]. Recall that $c(n, k)$ gives the cardinality of the set $\mathcal{S}_{n, k}$ of permutations of $[n]$ having exactly $k$ cycles, while $S(n, k)$ counts all partitions of $[n]$ having $k$ blocks. One may extend the bijective arguments of the prior section to provide combinatorial explanations of the following identities.

Proposition 3.3 If $n \geq 1$, then

$$
\sum_{k=1}^{n}(-1)^{k}\left(\begin{array}{c}
2 n+1 \\
n-k
\end{array}\right) c(n+k, k)=(-1)^{n}
$$

and

$$
\sum_{k=1}^{n}(-1)^{k}\left(\begin{array}{c}
2 n+1 \\
n-k
\end{array}\right) S(n+k, k)=(-1)^{n} n !
$$


Proof. Given $1 \leq k \leq n$, let $\mathcal{A}_{k}$ denote the set of all ordered pairs $\lambda=(A, B)$, where $A$ is a subset of $[2 n+1]$ of size $n-k$ and $B$ is a permutation of the $n+k$ smallest elements of $[2 n+1] \backslash A$ having $k$ cycles. Define the sign of $\lambda \in \mathcal{A}_{k}$ as $(-1)^{k}$ and let $\mathcal{A}=\cup_{k=1}^{n} \mathcal{A}_{k}$. Then the left side of (14) gives the sum of the signs of all members of $\mathcal{A}$. We define an involution on $\mathcal{A}$ as follows. First suppose $2 n+1 \in A$ within $\lambda=(A, B) \in \mathcal{A}$. Let $m$ be the largest element of $[2 n+1] \backslash A$. Let $\left\{s_{1}, s_{2}, \ldots, s_{n+k}\right\}$ denote the elements within the permutation $B$ in ascending order. We then let $\lambda^{\prime}=\left(A^{\prime}, B^{\prime}\right)$ be obtained from $\lambda$ as follows. We remove $2 n+1$ from $A$ to obtain $A^{\prime}$. For $B^{\prime}$, we first replace each element $s_{i}$ of $B$ with $s_{i+1}$ for $1 \leq i<n+k$ and replace the element $s_{n+k}$ with $m$. Then add $s_{1}$ as a 1 -cycle to the resulting permutation to obtain $B^{\prime}$. For example, if $n=7, k=4$ and $\lambda=(A, B) \in \mathcal{A}_{4}$ where $A=\{4,13,15\}$ and $B=(5,2,1),(9,3,6),(10,8),(12,7,11)$, then $\lambda^{\prime}=\left(A^{\prime}, B^{\prime}\right) \in \mathcal{A}_{5}$ where $A^{\prime}=\{4,13\}$ and $B^{\prime}=(1),(6,3,2),(10,5,7),(11,9),(14,8,12)$. Note that $\lambda \in \mathcal{A}_{k}$ implies $\lambda^{\prime} \in \mathcal{A}_{k+1}$, whence $\lambda$ and $\lambda^{\prime}$ have opposite sign. Conversely, if $2 n+1 \notin A$ and the smallest element within $B$ belongs to a cycle by itself, we reverse the prior procedure.

So assume that $2 n+1 \notin A$ (in which case $2 n+1$ belongs to neither $A$ nor $B$ ), and that the smallest element, say $b$, within $B$ does not belong to a cycle by itself. In this case, let $x_{0}$ be the largest element $x>b$ (if it exists) satisfying either (i) $x \in A$, or (ii) $x \in B$ and occurs as a 1-cycle in $B$. Replacing option (i) with (ii) concerning the element $x_{0}$, and vice-versa, changes the sign and is an involution where it is defined. For example, if $n=7$ and $k=4$, then $(A, B) \in \mathcal{A}_{4}$ where $A=\{1,6,8\}$ and $B=(7,3,4),(12,2,5,10,9),(13),(14,11)$ is paired with $(C, D) \in \mathcal{A}_{3}$ where $C=\{1,6,8,13\}$ and $D=(7,3,4),(12,2,5,10,9),(14,11)$ (note that $b=2$ and $\left.x_{0}=13\right)$. Combining this mapping with the previous one then defines a sign-changing involution of $\mathcal{A} \backslash \mathcal{A}^{*}$, where $\mathcal{A}^{*}$ consists of all pairs $(A, B)$ in which $A=[n-k]$ for some $1 \leq k \leq n$ and $B$ is a member of $\mathcal{S}_{n+k, k}$ (on the elements in $\{n-k+1, \ldots, 2 n\})$ containing no 1-cycles (i.e., is a derangement). Thus, the sum of the signs of all members of $\mathcal{A}^{*}$ is given by $\sum_{k=1}^{n}(-1)^{k} d(n+k, k)$. Applying the mapping of the previous section shows that each member of $\mathcal{A}^{*}$ is paired with another of opposite sign except for $\lambda=(A, B)$, where $A=\emptyset$ and $B=(2,1),(4,3), \ldots,(2 n, 2 n-1)$, which has sign $(-1)^{n}$. This completes the proof of (14).

The proof of (15) follows in a similar fashion, upon replacing permutations with partitions in the appropriate places. One obtains that the sum of signs of the set of survivors is given by $\sum_{k=1}^{n}(-1)^{k} e(n+$ $k, k)$. In the following section, a bijective proof showing that this sum equals $(-1)^{n} n$ ! is given, which implies (15).

Remark. Identities (14) and (15) correspond, respectively, to the $m=1$ cases of Theorems 17 and 18 from [5], which were shown by algebraic methods.

\section{Combinatorial proofs of identities (11) and (13)}

In this section, we provide bijective proofs of identities (11) and (13) above. We first prove (11), rewritten in the form

$$
\sum_{k=1}^{n}(-1)^{k} e(n+k, k)=(-1)^{n} n ! .
$$




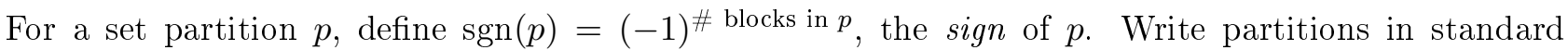
increasing form: entries in each block increasing, largest entries increasing left to right, as in

$$
23 / 158 / 4679 \text {. }
$$

Let $\mathcal{P}_{n, k}$ denote the set of singleton-free partitions of $[n+k]$ with $k$ blocks. Set $\mathcal{A}_{n}=\bigcup_{k=1}^{n} \mathcal{P}_{n, k}$, where each element of $\mathcal{A}_{n}$ has a sign as defined above. Thus $\sum_{p \in \mathcal{A}_{n}} \operatorname{sgn}(p)$ gives the left side of (16). We note that every nonempty singleton-free partition $p$ lies in a unique $\mathcal{A}_{n}$, namely, take $n \geq 1$ to be the total number of nonterminal entries in the blocks of $p$; we refer to $n$ as the size of $p$. Hence, we have

LEMMA $4.1 \mathcal{A}_{n}$ consists of the singleton-free partitions of size $n$.

To account for the $n$ ! on the right side of (16), we define a bad partition to be a pairing of the elements of $[n]$ with those in $[n+1,2 n]$ for some $n$, such as $24 / 15 / 36$ with $n=3$. Note that $n$ will then be the size (in the sense defined above) of the bad partition. Let $\mathcal{B}_{n}$ denote the set of bad partitions of size $n$. Clearly, $\mathcal{B}_{n} \subseteq \mathcal{P}_{n, n} \subseteq \mathcal{A}_{n}$ and $\left|\mathcal{B}_{n}\right|=n$ !.

Now set

$$
\mathcal{A}_{n}^{*}=\mathcal{A}_{n} \backslash \mathcal{B}_{n} .
$$

Since the partitions in $\mathcal{B}_{n}$ all have sign $(-1)^{n}$, the identity (16) is equivalent to

$$
\sum_{p \in \mathcal{A}_{n}^{*}} \operatorname{sgn}(p)=0
$$

To establish (18), we define a sign-reversing involution on $\mathcal{A}_{n}^{*}$, or equivalently, in view of Lemma 4.1, a size-preserving sign-reversing involution on the set of non-bad singleton-free partitions. The idea is to change the number of blocks by 1 by either deleting the last block and placing all its nonterminal entries in some earlier block or, when appropriate, transferring some entries from an existing block to form the nonterminal entries of a new last block.

It is helpful to introduce some terminology. For a singleton-free partition $p$ of size $n$, split each block into an upper segment consisting of the entries $\geq n+1$ and a lower segment consisting of the entries $\leq n$. Thus each block has two complementary segments. A segment $S$ is lonely if its complement, denoted $S^{\prime}$, is empty, that is, if $S$ constitutes the entire block. For example, the partition (17) has 6 nonterminal entries - so has size 6-and can be written in segmented form as

$$
8 \quad 79
$$

231546,

with upper segments in the top row. Here, 23 is the only lonely segment. In these terms, a singletonfree partition is bad when all its segments have length 1 . We order nonempty segments by largest entry and write $S \succ T$ if the max entry of $S$ is larger than the max entry of $T$. Thus $46 \succ 15 \succ 23$. From now on, we present partitions in segmented form. The following observation will be useful.

Lemma 4.2 Suppose $p$ is a singleton-free partition in segmented form. Then the number of elements in the top row is equal to the number of blocks. 
Proof. By construction, the elements in the bottom row are $1,2, \ldots, n$ where $n$ is the size of the partition. But, by definition, size is the total number of nonterminal entries in the blocks. So the number of elements in the top row must equal the number of terminal entries in the blocks, i.e., the number of blocks.

Now, here is a mapping that will turn out to be the desired involution. Given $p \in \mathcal{A}_{n}^{*}$, look for a nonempty upper segment $U$ such that (i) its complement $U^{\prime}$ contains at least 2 elements, and (ii) $U^{\prime} \succ L$ for all lonely lower segments (if any). If found, take the $U$ with largest $U^{\prime}$ (meaning largest with respect to $\succ$ ) and transfer the elements of $U$ from its block to create a new last block $U \cup\{1+\max (p)\}$. In (19), for instance, both upper segments work and $U=79$ has the largest $U^{\prime}$, leading to

$$
\begin{array}{cccc} 
& & 8 & 7910 \\
23 & 46 & 15 &
\end{array} \text {, }
$$

after standardizing the order of the blocks.

If there is no such $U$, look for a nonempty lower segment $L$ such that, analogously, (i) $L^{\prime}$ contains at least 2 elements and (ii) $L^{\prime} \succ U$ for all lonely upper segments. If found, take the $L$ with largest $L^{\prime}$ and transfer the elements of $L$ from its block to create a new last block $L \cup\{1+\max (p)\}$. For example,

$$
\begin{array}{cccc} 
& & 89 & 710 \\
24 & 35 & 1 & 6
\end{array}
$$

has no such $U$, but $L=1$ and $L=6$ both work, leading to

$$
\begin{array}{ccccc} 
& & 89 & 710 & 11 \\
24 & 35 & 1 & & 6
\end{array} .
$$

When such a $U$ or $L$ exists, we call this the lift operation and say that the original partition $p$ is liftable. Also, we call segments satisfying the conditions valid, formalized in

Definition 4.3 Suppose $p$ is a singleton-free partition. An upper segment $U$ is valid iff $U \neq \emptyset$, $\left|U^{\prime}\right| \geq 2$, and $U^{\prime} \succ L$ for all lonely lower segments $L$. A lower segment $L$ is valid iff $L \neq \emptyset,\left|L^{\prime}\right| \geq 2$, and $L^{\prime} \succ U$ for all lonely upper segments $U$.

Thus a singleton-free partition is liftable precisely when it has at least one valid segment. We note that a bad partition is never liftable; this is clear because, by definition, the complementary segment of a valid segment has length at least 2 .

For the remaining case ( $p$ not liftable), we need a lemma.

Lemma 4.4 Suppose $n \geq 1$ and $p$ is a singleton-free partition of size $n$, and suppose that $p$ is not liftable (no valid segments). Then

1. the set $S$ of nonterminal elements in the last block is lonely (that is, either an upper set, with all elements $\geq n+1$, or a lower set, with all elements $\leq n)$, and

2. unless $p$ is bad, there will exist a lonely segment $T$ at the opposite level (upper or lower) than $S$. 
Proof. If the set $S$ defined in item (1) is not lonely, then the last block has the segmented form $S_{1} m$ with $m$ the largest element of $p$ and $S_{1} \neq \emptyset, S_{2} \neq \emptyset$. But then the lower segment $S_{2}$ is clearly valid, a contradiction. So $S$ is lonely.

For item (2), suppose first that $S$ is upper (all elements $\geq n+1$ ) so that the upper segment of the last block contains at least 2 entries. We wish to show that there exists a lonely lower segment. But this is an immediate consequence of Lemma 4.2 (2 elements in one upper segment implies some other upper segment is empty).

Now suppose $S$ is lower and consider cases.

Case (i): Some upper segment $U$ has length $\geq 2$. If $U^{\prime}=\emptyset$, then $U$ is lonely and we are done. But if $U^{\prime} \neq \emptyset$, then some other upper segment is lonely (else $U^{\prime}$ would be valid).

Case (ii): All upper segments have length $\leq 1$. Lemma 4.2 then implies all upper segments have length exactly 1 and, in particular, there is neither an empty upper segment nor a lonely lower segment. If, now, some lower segment $L$ has length $\geq 2$, then $L^{\prime}$ is valid, a contradiction. This implies that all segments have length 1 and $p$ is a bad partition. The conclusion fails for bad partitions but they are excluded by hypothesis.

Now we complete the description of our mapping. If $p$ is not liftable, take the largest lonely segment $T$ of the type guaranteed by the second part of Lemma 4.4 and enlarge the block originally consisting of $T$ to $S \cup T$, and delete the last block. For example, for the singleton-free partitions (20) and (22), there is no valid segment, and the prescribed transfer leads back to the original partition in each case.

We have now defined a self-mapping, call it $\phi$, on the set of non-bad singleton-free partitions that preserves size (recall size $=$ total number of nonterminal entries) and always changes the number of blocks by 1 . It is clear from the construction that $\phi$ sends non-liftable partitions to liftable partitions and that $\phi^{2}$ is the identity on non-liftable partitions. The following lemma completes the proof that $\phi$ is an involution that interchanges the liftable and non-liftable partitions.

Lemma 4.5 If the partition $p$ is liftable, then $q:=\phi(p)$ is not liftable and $\phi(q)=p$.

Proof. Suppose first that $p$ has no valid upper segments so that a lower valid segment $L_{0}$ is lifted to get $\phi(p)$. Then $q$ has the form $\begin{array}{lllll}\ldots & U_{0} & \ldots & m \\ \ldots & \ldots & \ldots & L_{0}\end{array}$, where the dots represent undisturbed segments of $p$ and $m=\max (p)+1$. We proceed to show that no segment in $q$ is valid. This is clear for $U_{0}, \emptyset$ and $L_{0}$. Also, $m$ is not valid because, if it were, then its complement $L_{0}$ would have the properties necessary to make $U_{0}$ a valid upper segment in $p$, contrary to hypothesis. Apart from these four, any other valid segment in $q$ would already be valid in $p$. But $p$ has no valid upper segments, and if $L \neq L_{0}$ were a valid lower segment in $q$, then $L$ valid in $q$ implies $L^{\prime} \succ U_{0}$ since $U_{0}$ is lonely in $q$, while $L$ valid in $p$ implies $U_{0} \succ L^{\prime}$ by choice of the lifted segment $L_{0}$, yielding contradictory inequalities.

The proof for a lifted upper segment is similar and left to the reader. That $\phi(q)=p$ is now clear from the construction.

We conclude that $\phi$ is a sign-reversing involution on $\mathcal{A}_{n}^{*}$, as desired, which completes the proof of (11). We now prove identity (13) above by a bijective argument.

It is convenient to introduce lattice paths of upsteps $u=(1,1)$ and downsteps $d=(1,-1)$. Say such a path is balanced if it contains the same number of $u$ 's and $d$ 's so that it ends at ground level, and nonnegative if it never dips below ground level. Thus, a nonnegative balanced path is a Dyck path. Also, say a vertex is an ascent vertex if it is incident with two upsteps. Thus, the path $d d d u \cdot u \cdot u$ has 2 ascent vertices as indicated by the dots, and ududud has none. An AV-marked balanced (resp. Dyck) 
$n$-path is a balanced (resp. Dyck) path containing $n$ upsteps where some (all, or none) of the ascent vertices are distinguished ("marked"). Let $\mathcal{B}_{n}$ denote the set of $\mathrm{AV}$-marked balanced $n$-paths, and $\mathcal{B}_{n, k}$ the subset with $k$ marked ascent vertices. Thus $\mathcal{B}_{n}=\cup_{k=0}^{n-1} \mathcal{B}_{n, k}$. Define $\mathcal{D}_{n}$ and $\mathcal{D}_{n, k}$ analogously for Dyck paths. The cyclic rotation operator on balanced paths, $u^{i} d P \mapsto P d u^{i}$, is well known [8] to partition balanced $n$-paths into equivalence classes, all of size $n+1$, each of which contains exactly one Dyck path. Since it preserves ascent vertices and their marks, we see that $\left|\mathcal{D}_{n, k}\right|=\frac{1}{n+1}\left|\mathcal{B}_{n, k}\right|$.

Now let $\mathcal{F}_{n, k}$ denote the set of contents-ordered partitions of $[n+k]$ having $k$ blocks and no singletons, each with $\operatorname{sign}(-1)^{k}$, and set $\mathcal{F}_{n}=\cup_{k=1}^{n} \mathcal{F}_{n, k}$, so that $\left|\mathcal{F}_{n, k}\right|=f(n+k, k)$ and (13) becomes

$$
\sum_{p \in \mathcal{F}_{n}} \operatorname{sgn}(P)=(-1)^{n}(n+1) ! .
$$

There are a total of $n$ nonterminal entries in the blocks of a partition in $\mathcal{F}_{n, k}$. Replacing these entries by indistinguishable dots removes a factor of $n$ ! and reduces $\mathcal{F}_{n, k}$ to $\mathcal{F}_{n, k}^{*}:=\left\{(u, c): u=\left(u_{1}, \ldots, u_{k}\right)\right.$ is an increasing subsequence of length $k$ from $[n+k]$ and $c=\left(c_{1}, \ldots, c_{k}\right)$ is a $k$-part composition of $n\}$. (The $u_{i}$ are the last entries of the blocks, and $c_{i}$ is the number of nonterminal entries in the block containing $u_{i}$.) Here is a bijection from $\mathcal{F}_{n, k}^{*}$ to $\mathcal{B}_{n, n-k}$. Given $(u, c) \in \mathcal{F}_{n, k}^{*}$, form a path of $n+k$ steps with upsteps in positions $u_{1}, \ldots, u_{k}$. Then insert $c_{i}-1$ marked upsteps, $\boldsymbol{\bullet}$, after the $i$ th upstep, as illustrated in Figure 1 below for $u=(3,5,8,9), c=(1,2,1,3) \in \mathcal{F}_{7,4}^{*}$.

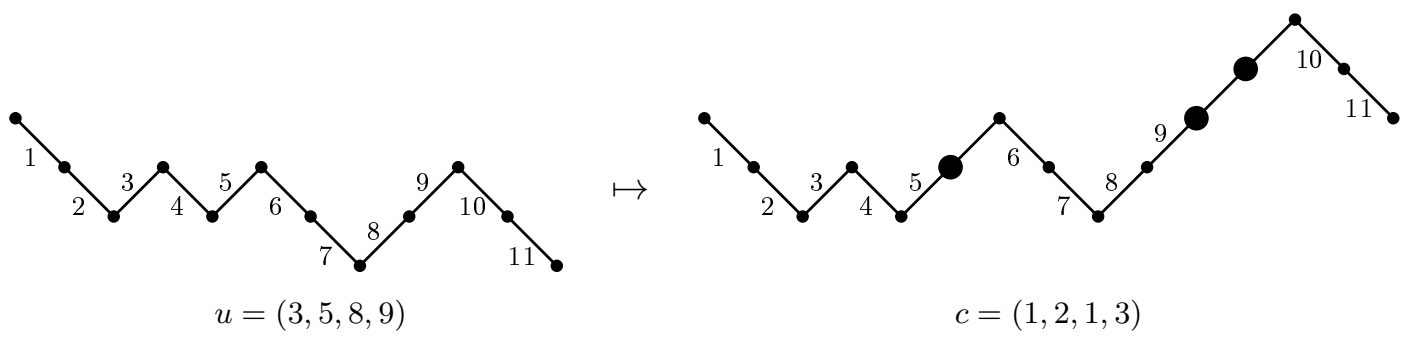

Figure 1: Example of mapping from $\mathcal{F}_{n, k}^{*}$ to $\mathcal{B}_{n, n-k}$ when $n=7$ and $k=4$.

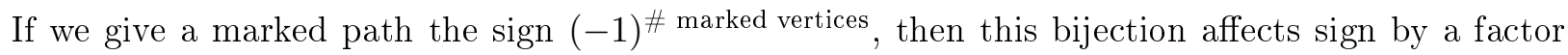
of $(-1)^{n}$ and so, after dividing first by $n$ ! and then by an additional $n+1$ as explained in the previous paragraph, (23) becomes

$$
\sum_{P \in \mathcal{D}_{n}} \operatorname{sgn}(P)=1
$$

The "sawtooth" path $(u d)^{n}$ has sign 1 and is the only element of $\mathcal{D}_{n}$ with no ascent vertices. Now, the map "flip the mark status of the first ascent vertex" is a sign-reversing involution on the rest of $\mathcal{D}_{n}$, and (24) follows.

\section{References}

[1] A. T. Benjamin and J. J. Quinn, Proofs that Really Count: The Art of Combinatorial Proof, Mathematical Association of America, Washington DC, 2003. 
[2] A. T. Benjamin And J. J. Quinn, An alternate approach to alternating sums: a method to die for, College Math. J., 39 (2008) 191-201.

[3] L. CARLitz, Note on the numbers of Jordan and Ward, Duke Math. J., 38 (1971) 783-790.

[4] L. Clark, Asymptotic normality of the Ward numbers, Discrete Math., 203 (1999) 41-48.

[5] H. W. Gould, H. Kwong and J. Quaintance, On certain sums of Stirling numbers with binomial coefficients, J. Integer Seq., 18 (2015) Art. 15.9.6.

[6] R. L. Graham, D. E. Knuth and O. Patashnik, Concrete Mathematics: A Foundation for Computer Science, Addison-Wesley, Boston, 1994.

[7] T. Mansour And M. Schork, Commutation Relations, Normal Ordering and Stirling Numbers, Chapman \& Hall/CRC an imprint of Taylor \& Francis LLC, 2015.

[8] T. V. Narayana, Cyclic permutation of lattice paths and the Chung-Feller theorem, Skand. Aktuarietidskr, 1967 (1967) 23-30.

[9] N. J. A. Sloane, On-Line Encyclopedia of Integer Sequences, at http://oeis.org.

[10] R. P. Stanley, Enumerative Combinatorics, Vol. I, Cambridge University Press, 1997.

[11] M. WARD, The representation of Stirling's numbers and Stirling's polynomials as sums of factorials, Amer. J. Math., 56 (1934) 87-95. 